\title{
Prognosis and clinicopathological characteristics of renal cell carcinoma: does bilateral occurrence influence overall and cancer-specific survival?
}

\author{
Tao Jiang", Yu-Peng Wu", Shao-Hao Chen", Zhi-Bin Ke, Ying-Chun Liang, Ning Xu \\ Department of Urology, First Affiliated Hospital of Fujian Medical University, Fuzhou 350005, China \\ Contributions: (I) Conception and design: N Xu, YP Wu; (II) Administrative support: N Xu; (III) Provision of study materials or patients: None; \\ (IV) Collection and assembly of data: T Jiang, SH Chen, YC Liang; (V) Data analysis and interpretation: T Jiang, YP Wu, SH Chen, ZB Ke; \\ (VI) Manuscript writing: All authors; (VII) Final approval of manuscript: All authors. \\ \#These authors contributed equally to this work. \\ Correspondence to: Ning Xu. Department of Urology, First Affiliated Hospital of Fujian Medical University, 20 Chazhong Road, Fuzhou 350005, \\ China. Email: drxun@fjmu.edu.cn.
}

Background: To compare clinicopathological characteristics and prognosis of bilateral and unilateral renal cell carcinoma.

Methods: Data from patients who had been diagnosed with renal cell carcinoma (RCC) and undergone radical nephrectomy (RN) from 2004 to 2014 were extracted from the Surveillance, Epidemiology, and End Results (SEER) registry. The $\chi^{2}$ test was used to compare relevant characteristics between patients with bilateral and unilateral RCC. Survival curves were generated by the Kaplan-Meier method and the log-rank test was used to compare overall survival (OS) and cancer-specific survival (CSS). Cox proportional hazards regression analysis was used to determine the risk factors for OS and CSS.

Results: The study cohort comprised 41,573 patients. A nonlinear relationship between age and OS and CSS was identified with and without adjusting for potential factors. Threshold effect analysis revealed that ages 54 and age 74 were the turning points associated with changes in OS and CSS, respectively. Multivariate Cox regression analysis demonstrated that age category, race, grade, and T, N, and M stage were prognostic factors for OS and CSS of patients with RCC. Additionally, sex and pathology were significantly associated with OS.

Conclusions: Bilateral occurrence does not influence OS and CSS in patients with RCC who have undergone RN. The risk of poor OS and CSS was higher with greater age category, tumor grade, and T stage. Patients in different age categories $(<54, \geq 54$ and $<74, \geq 74$ years) may benefit from individualized attention and therapeutic strategies.

Keywords: Unilateral; bilateral; renal cell carcinoma (RCC); overall survival (OS); cancer-specific survival (CSS)

Submitted Apr 04, 2019. Accepted for publication Oct 11, 2019.

doi: $10.21037 /$ tcr.2019.11.22

View this article at: http://dx.doi.org/10.21037/tcr.2019.11.22

\section{Introduction}

Renal cell carcinoma (RCC) is the most common type of kidney cancer in adults (1). The incidence of bilateral RCC varied from $1 \%$ to $5 \%$ of all patients with RCC $(2,3)$. Approximately $25 \%$ of patients with unilateral RCC reportedly develop severe preoperative renal dysfunction
$(4,5)$. Lowrance et al. (2) hypothesized that patients with bilateral RCC have more severe preoperative renal dysfunction than those with unilateral RCC. Balancing preservation of renal function and oncological efficacy is a challenge for urologists managing individuals with bilateral RCC (6). 
Identifying the differences between bilateral and unilateral RCC is important in facilitating provision of more effective therapeutic strategies and making a definitive and timely diagnosis in patients with bilateral RCC. Klatte et al. (7) reported that patients with bilateral RCC had Fuhrman grade 2 more often and Fuhrman grade 1 less often than those with unilateral RCC. Qi et al. (3) reported that the prognoses of patients with unilateral and bilateral RCC are comparable, which conflicts with Lowrance et al.'s conclusions (2). Thus, whether bilateral RCC adversely affects survival outcomes compared with unilateral RCC is controversial.

Because few studies have focused on the differences between bilateral and unilateral RCC, we evaluated the clinicopathological features and prognoses of these two groups in a large cohort.

\section{Methods}

\section{Statement of ethics approval}

SEER*Stat version 8.3.4 was used to generate a case listing. All information from the SEER database has been deidentified. Informed consent is not required for use of SEER data.

\section{Patients}

Inclusion criteria were as follows: year of diagnosis from 2004 to 2014; RCC the first and only cancer diagnosis; had undergone radical nephrectomy (patients with bilateral RCC to have undergone left- or right-sided radical nephrectomy); pathologically confirmed papillary renal cell carcinoma [not otherwise specified (ICD-O-3 $8260 / 3)$ ], or clear cell carcinoma renal cell carcinoma [not otherwise specified (ICD-O-3 8310/3)], or renal cell carcinoma [(ICD-O-3 8312/3)]. Patients with insufficient documentation of age at diagnosis, sex, or race/ethnicity were excluded from this study. Patients who were diagnosed with bladder cancer at autopsy only or at death, and those with other first primary cancers were also excluded. Duration of follow-up was calculated from 1 January 2004 to 31 December 2014.

\section{Statistical analysis}

The $\chi^{2}$ test was used to compare relevant characteristics between the RCC and unilateral RCC groups. Survival curves were generated by the Kaplan-Meier method and the log-rank test was used to compare overall survival (OS) and cancer-specific survival (CSS). Cox proportional hazards regression analysis was used to determine the risk factors for OS and CSS. OS was defined as the time from the date of diagnosis of RCC to the date of death from any cause. OS was censored at the date of last follow-up or 31 December 2014 for patients who were still alive. CSS was defined as the time from the date of diagnosis to the date of death from RCC. Data on patients who died of causes other than RCC were censored at the time of their death.

The relationships between age and OS and CSS after $\mathrm{RN}$ were explored by constructing smoothing plots. A twopiecewise linear regression model was used to examine the threshold effect of age on OS and CSS according to the smoothing plot. Tests for linear trend were performed by entering the median values for age, and $\mathrm{T}, \mathrm{N}$, and $\mathrm{M}$ stage as continuous variables in the modes. Statistical analyses were performed utilizing $\mathrm{R}$, version 3.4.1. Two-sided $\mathrm{P}<0.05$ was considered to denote statistical significance.

\section{Results}

\section{Patient characteristics}

A total of 41,573 patients met the eligibility criteria in this study, including 41,542 with unilateral and 31 with bilateral RCC. Relevant patient characteristics were stratified by laterality (Table 1). There were significant differences between the groups in sex, race, and $\mathrm{T}, \mathrm{N}$, and $\mathrm{M}$ stage, but no significant differences in age category, grade, or tumor pathology. Compared with patients with unilateral RCC, there was a greater proportion of male patients with bilateral RCC (male, $63.478 \%$ vs. $80.645 \%$, respectively; $\mathrm{P}=0.047)$. There was a higher portion of individuals classified as white in the unilateral than the bilateral RCC group (white, $88.634 \%$ vs. black, $75.862 \%$, respectively; $\mathrm{P}=0.040)$. Compared with patients with unilateral RCC, those with bilateral RCC had higher T stage (T3, 48.387\% vs. $27.902 \%$ and $\mathrm{T} 4,16.129 \%$ vs. $1.100 \%$, respectively; $\mathrm{P}<0.001)$, higher $\mathrm{N}$ stage (N1, $13.333 \%$ vs. $2.788 \%$, respectively; $\mathrm{P}=0.009)$, and higher $\mathrm{M}$ stage ( $\mathrm{M} 1,29.032 \%$ vs. $8.615 \%$, respectively; $\mathrm{P}<0.001$ ).

\section{Nonlinear relationship between age and OS and CSS}

A nonlinear relationship between age and OS (Figure 1A, Table 2) and CSS (Figure 1B, Table 2) was identified. The risk of poor OS increased with age to the turning point of 54 years (HR 1.045, 95\% CI: 1.038-1.052; $\mathrm{P}<0.001$ ), after 
Table 1 Relevant patient characteristics stratified by laterality

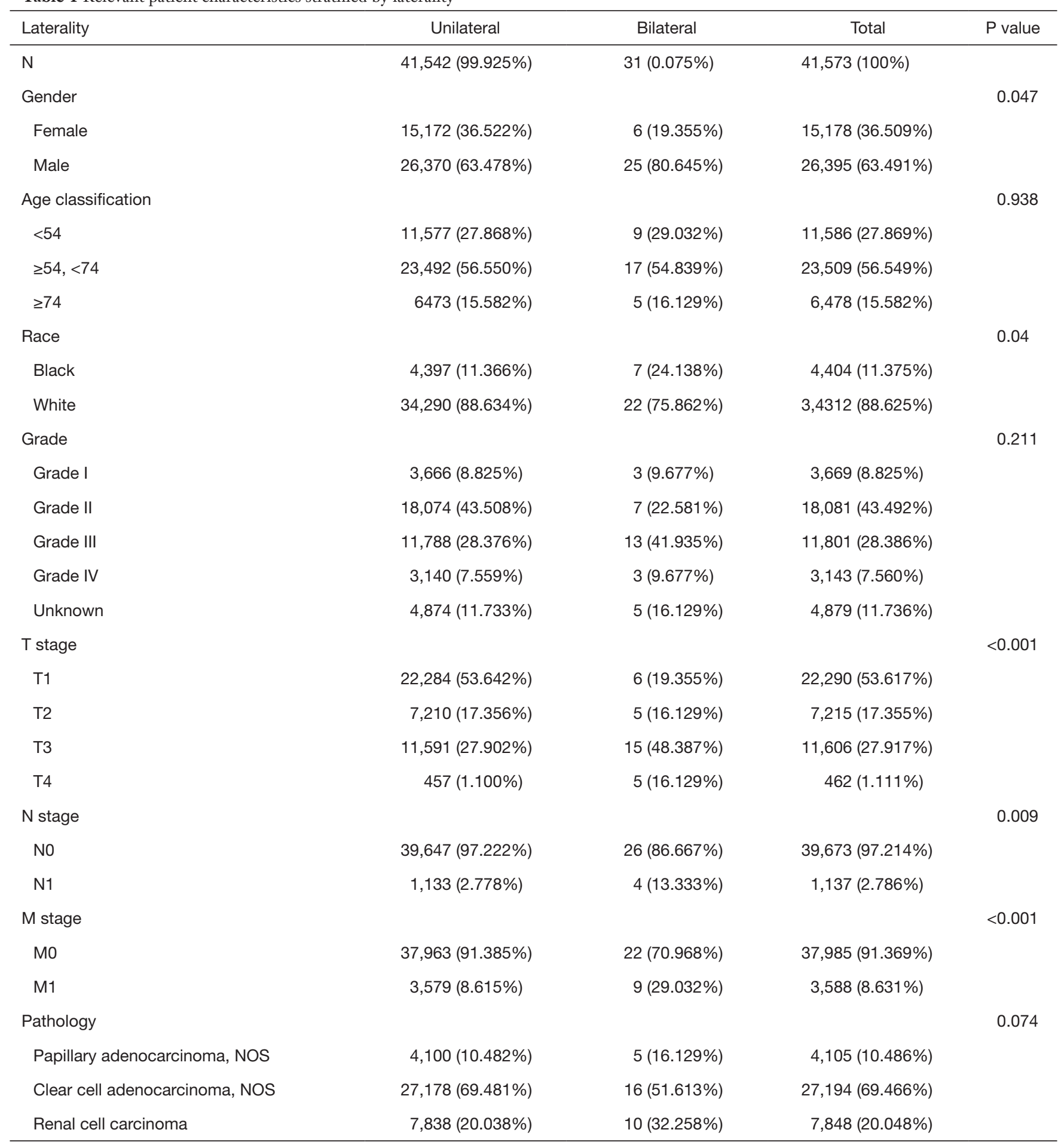

which the risk of poor OS decreased slightly with age from 54 years to a second turning point at 74 years (HR 1.031, 95\% CI: $1.028-1.034 ; \mathrm{P}<0.001)$. Thereafter, the risk of poor
OS again increased with age (HR 1.082, 95\% CI: $1.077-$ 1.088; $\mathrm{P}<0.001)$. The risk of poor CSS also increased with the age to the turning point of 54 years (HR 1.046, $95 \%$ 

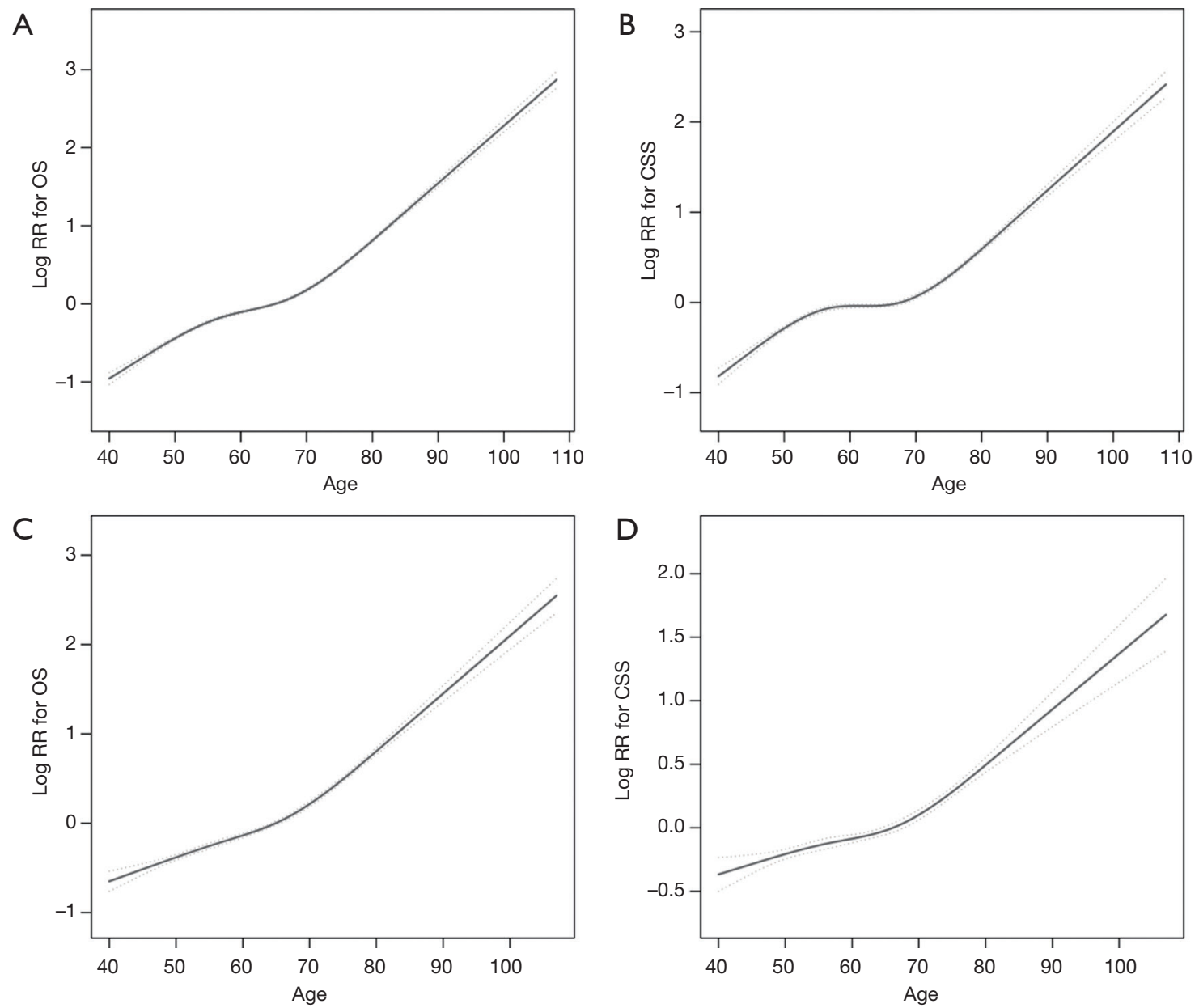

Figure 1 Relationships between age and overall survival (OS) and cancer-specific survival (CSS). A nonlinear relationship between age and OS (A) and CSS (B) was identified and was still present after adjusting for factors potentially associated with OS (C) and CSS (D), including laterality, sex, tumor pathology, race, grade, and T, N, and M stages.

CI: 1.037-1.054; $\mathrm{P}<0.001)$, after which the risk of poor CSS also decreased slightly to a second turning point of 74 years (HR 1.015, 95\% CI: 1.011-1.018; $\mathrm{P}<0.001)$. Thereafter, the risk of poor CSS again increased with age (HR 1.078, 95\% CI: $1.071-1.086$; $\mathrm{P}<0.001$ ).

After adjusting for factors potentially associated with OS and CSS, including laterality, sex, tumor pathology, race, grade, and $\mathrm{T}, \mathrm{N}$, and $\mathrm{M}$ stage, nonlinear relationships between age and OS (Figure 1C, Table 2) and CSS (Figure 1D, Table 2) were identified. However, the risk of poor OS and CSS did not decrease in patients aged from 54 to 74 years.

\section{Prognostic factors for OS and CSS of patients with RCC}

Unilateral Cox regression analysis revealed that laterality, sex, age category, race, grade, $T, N$, and $M$ stage, and tumor pathology were prognostic factors for CSS and OS in patients with RCC. Multivariate Cox regression analysis revealed that laterality was not associated with OS or CSS of patients with RCC whereas age category, race, grade, and $\mathrm{T}, \mathrm{N}$, and $\mathrm{M}$ stage were identified as prognostic factors for OS and CSS (Figure 2). However, only sex and tumor pathology were significantly associated with CSS of RCC patients according to multivariate Cox regression analysis. Increasing age was significantly associated with higher risk for both OS ( $\mathrm{P}$ for trend $<0.001$ ) and CSS ( $\mathrm{P}$ for trend $<0.001)$. Furthermore, higher tumor grade was significantly associated with higher risk for both OS (P for trend $<0.001$ ) and CSS ( $\mathrm{P}$ for trend $<0.001$ ). In addition, the higher tumor $\mathrm{T}$ stage was also significantly associated with higher risk for 
Table 2 Threshold effect analysis of age and overall survival and cancer-specific survival using a piecewise linear regression model

\begin{tabular}{lcccc}
\hline Turning point of age & CSS & CSS adjusted & OS & OS adjusted \\
\hline$<54$ & $1.046(1.037,1.054)^{\star}$ & $1.012(1.000,1.024)$ & $1.045(1.038,1.052)^{\star}$ & $1.020(1.010,1.030)^{\star}$ \\
$\geq 54,<74$ & $1.015(1.011,1.018)^{\star}$ & $1.018(1.012,1.023)^{\star}$ & $1.031(1.028,1.034)^{\star}$ & $1.034(1.030,1.039)^{\star}$ \\
$\geq 74$ & $1.078(1.071,1.086)^{\star}$ & $1.061(1.044,1.079)^{\star}$ & $1.082(1.077,1.088)^{\star}$ & $1.074(1.063,1.086)^{\star}$ \\
\hline
\end{tabular}

${ }^{\star} \mathrm{P}<0.001$. Adjusted: laterality, gender, pathology, race, grade, T stage, $\mathrm{N}$ stage, $\mathrm{M}$ stage. CSS, cancer-specific survival; OS, overall survival.
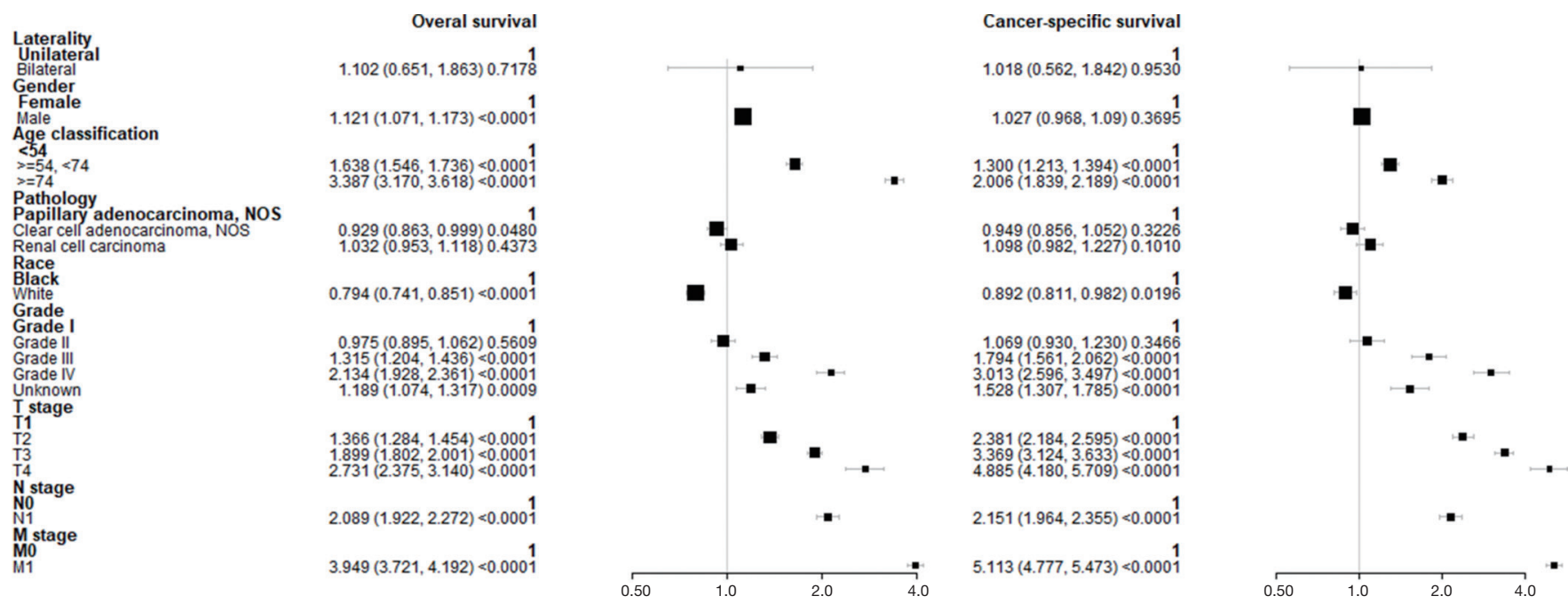

Figure 2 Multivariate Cox regression analysis of risk factors associated with overall survival and cancer-specific survival of RCC was performed and forest plots of hazard ratios generated.

both OS (P for trend $<0.001)$ and CSS (P for trend $<0.001)$.

\section{Comparison of survival stratified by laterality, age, grade, and T stage between unilateral and bilateral RCC patients}

Differences in OS (Figure $3 A$ ) and CSS (Figure 3B) according to laterality were assessed using Kaplan-Meier analysis, as were differences in OS and CSS stratified by age, grade, and $\mathrm{T}$ stage between patients with unilateral and bilateral RCC. Although patients with bilateral RCC had worse OS and CSS than those with unilateral RCC $(\mathrm{P}<0.001)$, multivariate Cox regression analysis demonstrated that laterality was not an independent prognostic factor for OS or CSS. Differences in OS stratified by age and $\mathrm{T}$ stage were identified, as shown in Figures 4 and 5A, respectively. Differences in CSS stratified by age and $\mathrm{T}$ stage were also identified, as shown in Figures $4 B$ and Figure 5B, respectively.

\section{Subgroup analysis of OS and CSS stratified by laterality}

Multivariate Cox regression analysis revealed that laterality was not associated with OS or CSS in patients with RCC. We therefore examined associations with other confounders using multivariate Cox regression and survival stratified by laterality (Figure 6). As shown in Figure 6, there were no significant differences in OS and CSS for age $\geq 74$ years, female sex, T1, T4, N1, or M1 when hazard ratios for unilateral and bilateral RCC were determined, suggesting that these variables are confounding factors of laterality.

\section{Discussion}

In this study, we investigated of survival outcomes in patients with bilateral and unilateral RCC. The main finding was that these two patient groups have equivalent OS and CSS. Age category, race, grade, and T, N, and M stage were significant prognostic factors for OS and CSS 
A
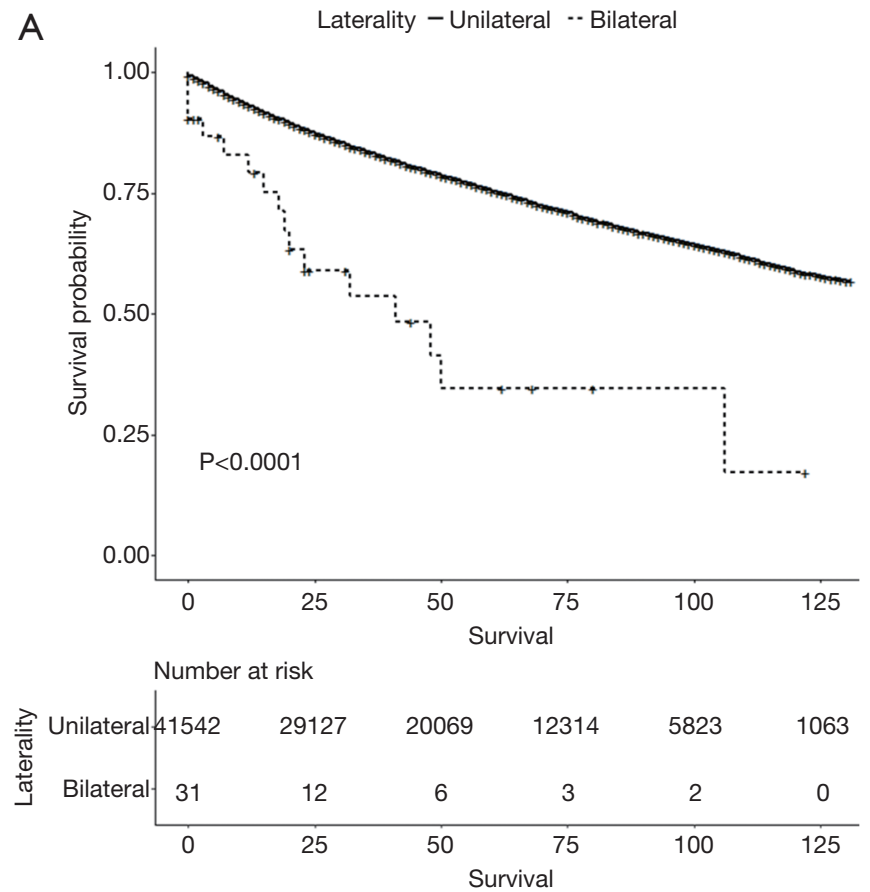
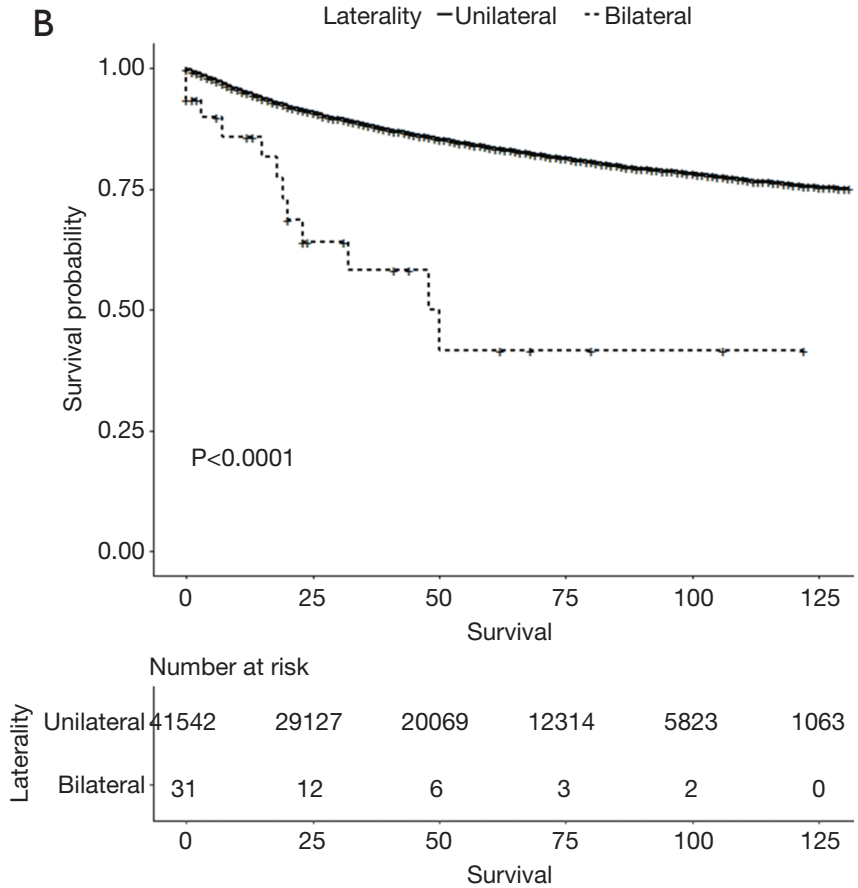

Figure 3 Overall survival (A) and cancer specific survival (B) of patients with renal cell carcinoma according to laterality.
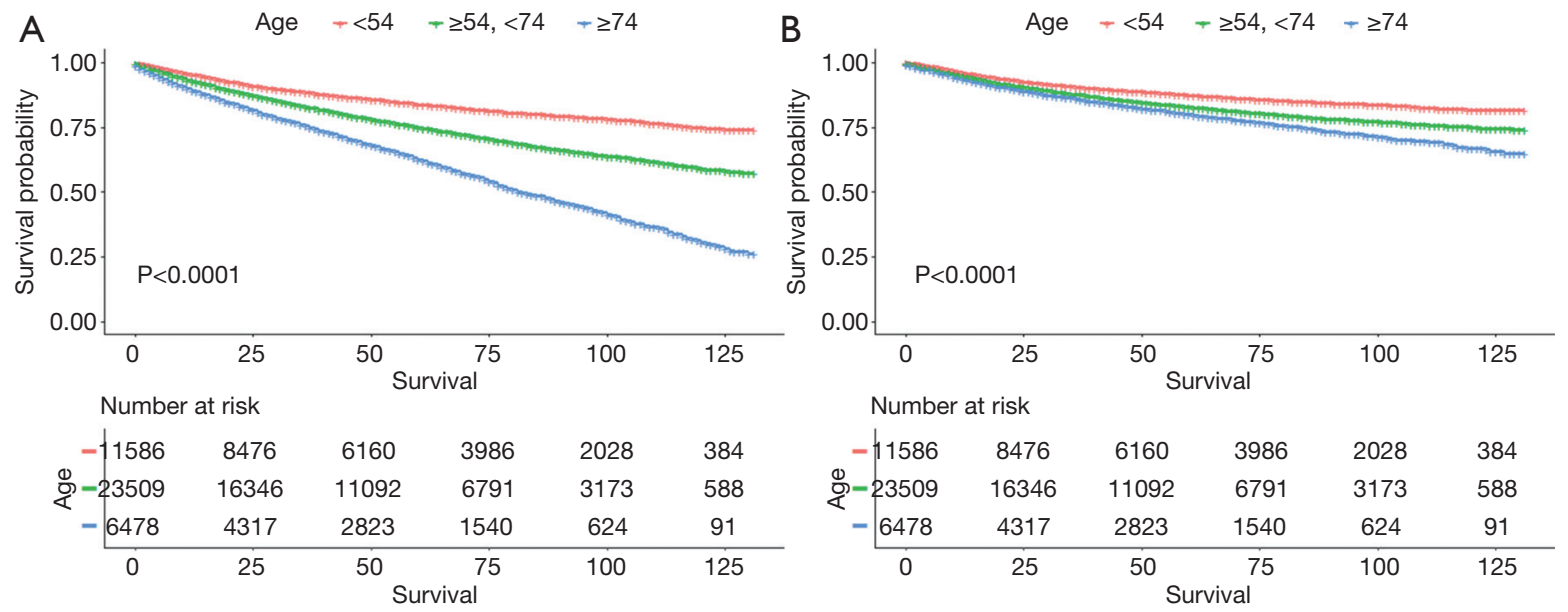

Figure 4 Overall survival (A) and cancer specific survival (B) of patients with renal cell carcinoma according to age category.

in all patients with RCC. Additionally, sex and tumor pathology were significantly associated with OS in these patients.

In this study, we identified a nonlinear relationship between age and OS and CSS. Two turning points in age (54 and 74 years) were identified by threshold effect analysis.

To validate the effects of these two age turning points, we added age category to the multivariate Cox regression model and then identified that age category was significantly associated with OS and CSS of the study patients. Several studies have already investigated the effects of age at diagnosis of RCC. However, most of these studies focused on the young adults (8-10) and very few investigated analyzed in older individuals (11-13). The studies cited above mainly focused on clinical and pathologic variables in distinct age categories. In this study, we looked for a distinct 

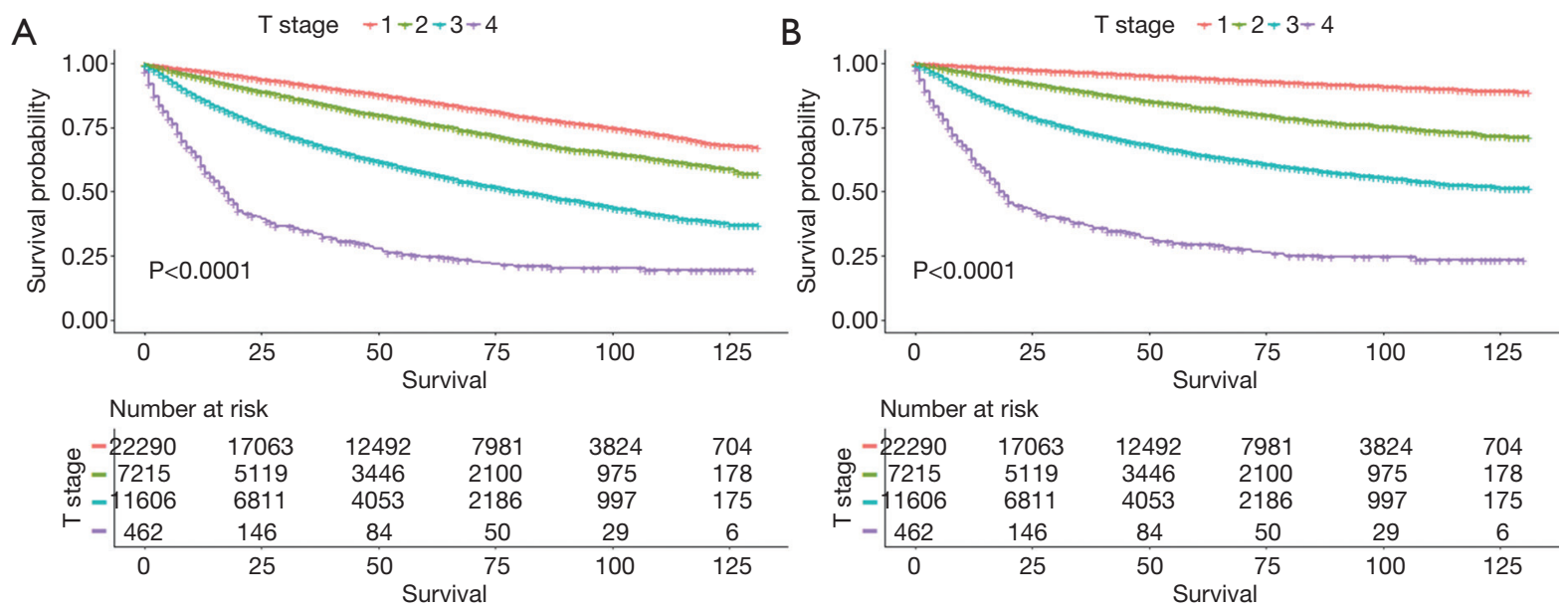

Figure 5 Overall survival (A) and cancer specific survival (B) of patients with renal cell carcinoma according to $\mathrm{T}$ stage.
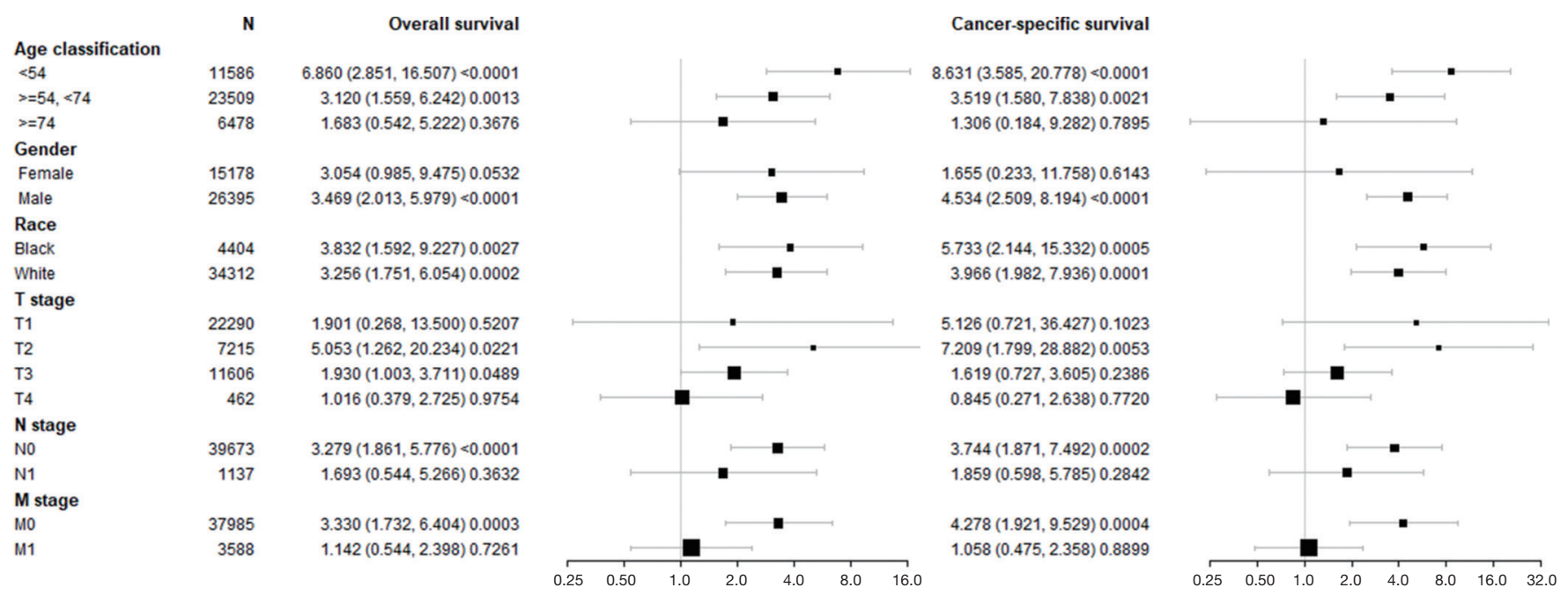

Figure 6 Subgroup analysis was performed to determine potential confounding factors of laterality and forest plots of hazard ratio generated.

turning point in age in a cohort of patients with RCC of all ages. Verhoest et al. (13) reported that the maximum rate of incidental RCC occurs between 60 and 80 years of age. A potential explanation for the slightly lower risk of poor OS and CSS in the 54 to 74 years age group than in the under 54 and over 74 years age groups is that patients in the 54 to 74 years age group may undergo more radiologic investigations for various comorbidities and thus receive more timely treatment.

Most RCCs are unilateral, bilateral RCCs occurring more frequently in patients with hereditary RCC syndromes (3). Although the prognoses of unilateral and bilateral RCC have been compared in several studies, they are still controversial. Simmons et al. (14) reported 5-year-OS rates of $86 \%$ and 10 -year-OS rates of $71 \%$ in 220 patients with bilateral RCC. Several groups (15-18) have reported 5-yearCSS as $80 \%$ to $87 \%$ and 10 -year-CSS from $70 \%$ to $82 \%$ in patients with bilateral RCC. In this study, multivariate analysis demonstrated that patients with bilateral RCC had comparable oncological outcomes to those with unilateral RCC, which is consistent with the studies cited above.

Additionally, Volpe et al. (19) reported that TNM stage is the strongest independent prognostic factor for localized RCC. In this study, multivariate Cox regression analysis also indicated that TNM stage is the strongest risk factor for OS and CSS in patients with RCC. It is well known that higher 
$\mathrm{T}, \mathrm{N}$, and $\mathrm{M}$ stages are associated with worse prognoses and shorter OS and CSS (20-22). Sex has also been established as an independent risk factor for patients with RCC (23). In this study, male patients were at a 1.121-fold higher risk of poor OS than female patients, which is consistent with previous studies $(24,25)$. However, there was no significant difference between male and female patients in terms of poor CSS. Berndt et al. (26) have reported that OS is poorer in black than in white individuals after adjustment for demographic variables and prognostic factors; these findings are consistent with those of the present study. Another study of 39,432 patients with RCC also identified a poorer OS in black than in whites individuals (23). Schrader et al. (27) found that the long term prognoses of papillary RCC and clear cell RCC are comparable. Waalkes et al. (28) reported that papillary RCC has a significantly better prognosis than clear cell RCC, which is inconsistent with our findings; there was no significant difference in pathological classification.

This study has some limitations. First, bilateral RCC can develop synchronously or metachronously, and there is evidence that multifocality is more common in sporadic bilateral RCC than in unilateral RCC. Siemer et al. (29) reported that the prognosis of patients with metachronous bilateral RCC is poorer. In contrast, BoorJian et al. (15) found no difference between metachronous and synchronous bilateral RCC. Several studies $(7,30)$ have also found that multifocality is not associated with survival outcomes in patients with RCC. Because information about synchronicity is not available from the SEER database, we were unable to include this variable in our multivariate Cox regression model of OS and CSS. Second, bilateral RCC occurs more frequently in patients with hereditary RCC syndromes than in those with sporadic RCC. Because these variables are also not obtainable from the SEER database, we could not investigate them. Additionally, because of its retrospective nature, the study has selection bias and lacks randomization, the effects of which we minimized by performing subgroup analyses to determine potential confounding factors of laterality, thus strengthening the statistical power of our analysis of risk factors.

\section{Conclusions}

Bilaterality does not influence OS or CSS of patients with RCC who have undergone RN. The risk of poor OS and CSS is associated with increasing age category, tumor grade, and $\mathrm{T}$ stage. Patients in the age categories of $<54,54$ to
$<74$, and $\geq 74$ years) may require individualized assessment and therapeutic strategies.

\section{Acknowledgments}

Funding: None.

\section{Footnote}

Conflicts of Interest: All authors have completed the ICMJE uniform disclosure form (available at http://dx.doi. org/10.21037/tcr.2019.11.22). The authors have no conflicts of interest to declare

Ethical Statement: The authors are accountable for all aspects of the work in ensuring that questions related to the accuracy or integrity of any part of the work are appropriately investigated and resolved.

Open Access Statement: This is an Open Access article distributed in accordance with the Creative Commons Attribution-NonCommercial-NoDerivs 4.0 International License (CC BY-NC-ND 4.0), which permits the noncommercial replication and distribution of the article with the strict proviso that no changes or edits are made and the original work is properly cited (including links to both the formal publication through the relevant DOI and the license). See: https://creativecommons.org/licenses/by-nc-nd/4.0/.

\section{References}

1. Chen SH, Wu YP, Li XD, et al. R.E.N.A.L. Nephrometry Score: A Preoperative Risk Factor Predicting the Fuhrman Grade of Clear-Cell Renal Carcinoma. J Cancer 2017;8:3725-32.

2. Lowrance WT, Yee DS, Maschino AC, et al. Developments in the surgical management of sporadic synchronous bilateral renal tumours. BJU Int 2010;105:1093-7.

3. Qi N, Li T, Ning X, et al. Clinicopathologic Features and Prognosis of Sporadic Bilateral Renal Cell Carcinoma: A Series of 148 Cases. Clin Genitourin Cancer 2017;15:618-24.

4. Donin NM, Suh LK, Barlow L, et al. Tumour diameter and decreased preoperative estimated glomerular filtration rate are independently correlated in patients with renal cell carcinoma. BJU Int 2012;109:379-83.

5. Russo P, Mano R. Open mini-flank partial nephrectomy: an essential contemporary operation. Korean J Urol 
2014;55:557-67.

6. Zhang S, Zhao X, Ji C, et al. Radiofrequency ablation of synchronous bilateral renal cell carcinoma. Int J Urol 2012;19:241-7.

7. Klatte T, Wunderlich H, Patard JJ, et al. Clinicopathological features and prognosis of synchronous bilateral renal cell carcinoma: an international multicentre experience. BJU Int 2007;100:21-5.

8. Eggener SE, Rubenstein JN, Smith ND, et al. Renal tumors in young adults. J Urol 2004;171:106-10.

9. Sánchezortiz RF, Rosser CJ, Madsen LT, et al. Young age is an independent prognostic factor for survival of sporadic renal cell carcinoma. J Urol 2004;171:2160-5.

10. Hi AEF, Cherullo EE, El-Jack M, et al. Sporadic renal cell carcinoma in young adults: presentation, treatment, and outcome. Urology 2002;60:806-10.

11. Bensalah K, Sadiq A, Guillé F, et al. Risks and benefits of total nephrectomy in elderly patients over the age of 80 . Prog Urol 2005;15:632-5.

12. Cohen HT, Mcgovern FJ. Renal-cell carcinoma. N Engl J Med 2005;353:2477-90.

13. Verhoest G, Veillard D, Guillé F, et al. Relationship between age at diagnosis and clinicopathologic features of renal cell carcinoma. Eur Urol 2007;51:1298-305.

14. Simmons MN, Brandina R, Hernandez AF, et al. Surgical Management of Bilateral Synchronous Kidney Tumors: Functional and Oncological Outcomes. J Urol 2010;184:865-72.

15. Boorjian SA, Crispen PL, Lohse CM, et al. The Impact of Temporal Presentation on Clinical and Pathological Outcomes for Patients with Sporadic Bilateral Renal Masses. Eur Urol 2008;54:855-65.

16. Blute ML, Itano NB, Cheville JC, et al. The effect of bilaterality, pathological features and surgical outcome in nonhereditary renal cell carcinoma. J Urol 2003;169:1276-81.

17. Pahernik S, Cudovic D, Roos F, et al. Bilateral synchronous sporadic renal cell carcinoma: surgical management, oncological and functional outcomes. BJU Int 2007;100:26-9.

18. Becker F, Siemer S, A, Suttmann H, et al. Long-term survival in bilateral renal cell carcinoma: a retrospective single-institutional analysis of 101 patients after surgical treatment. Urology 2008;72:349-53.

19. Volpe A, Patard JJ. Prognostic factors in renal cell carcinoma. World J Urol 2010;28:319-27.
20. Finley DS, Pantuck AJ, Belldegrun AS. Tumor biology and prognostic factors in renal cell carcinoma. Oncologist 2011;16 Suppl 2:4-13.

21. Delahunt B, Kittelson JM, McCredie MR, et al. Prognostic importance of tumor size for localized conventional (clear cell) renal cell carcinoma: assessment of TNM T1 and T2 tumor categories and comparison with other prognostic parameters. Cancer 2002;94:658-64.

22. Moch H, Gasser T, Amin MB, et al. Prognostic utility of the recently recommended histologic classification and revised TNM staging system of renal cell carcinoma: a Swiss experience with 588 tumors. Cancer 2000;89:604-14.

23. Stafford HS, Saltzstein SL, Shimasaki S, et al. Racial/ ethnic and gender disparities in renal cell carcinoma incidence and survival. J Urol 2008;179:1704-8.

24. Neuzillet Y, Tillou X, Mathieu R, et al. Renal cell carcinoma (RCC) in patients with end-stage renal disease exhibits many favourable clinical, pathologic, and outcome features compared with RCC in the general population. Eur Urol 2011;60:366-73.

25. Hurst FP, Jindal RM, Fletcher JJ, et al. Incidence, predictors and associated outcomes of renal cell carcinoma in long-term dialysis patients. Urology 2011;77:1271-6.

26. Berndt SI, Carter HB, Schoenberg MP, et al. Disparities in treatment and outcome for renal cell cancer among older black and white patients. J Clin Oncol 2007;25:3589-95.

27. Schrader AJ, Rauer-Bruening S, Olbert PJ, et al. Incidence and long-term prognosis of papillary renal cell carcinoma. J Cancer Res Clin Oncol 2009;135:799-805.

28. Waalkes S, Roos FC, Eggers H, et al. Incidence and longterm prognosis of papillary renal cancer. Results of a retrospective multicenter study. Urologe A 2011;50:1125-9.

29. Siemer S, Uder M, Zell A, et al. Bilateral kidney tumor. Therapy management and histopathological results with long-term follow-up of 66 patients. Urologe A 2001;40:114-20.

30. Siracusano S, Novara G, Antonelli A, et al. Prognostic role of tumour multifocality in renal cell carcinoma. BJU Int 2012;110:E443-8.

Cite this article as: Jiang T, Wu YP, Chen SH, Ke ZB, Liang $\mathrm{YC}, \mathrm{Xu}$ N. Prognosis and clinicopathological characteristics of renal cell carcinoma: does bilateral occurrence influence overall and cancer-specific survival? Transl Cancer Res 2020;9(2):432440. doi: 10.21037/tcr.2019.11.22 